\title{
Deflection of ferromagnetic and antiferromagnetic skyrmions at heterochiral interfaces
}

\author{
Raí M. Menezes, ${ }^{1,2}$ Jeroen Mulkers, ${ }^{1,3}$ Clécio C. de Souza Silva, ${ }^{2}$ and Milorad V. Milošević1 ${ }^{1, *}$ \\ ${ }^{1}$ Departement Fysica, Universiteit Antwerpen, Groenenborgerlaan 171, B-2020 Antwerpen, Belgium \\ ${ }^{2}$ Departamento de Física, Universidade Federal de Pernambuco, Cidade Universitária, 50670-901, Recife-PE, Brazil \\ ${ }^{3}$ DyNaMat Lab, Department of Solid State Sciences, Ghent University, B-9000 Ghent, Belgium
}

(Received 16 November 2018; revised manuscript received 18 January 2019; published 7 March 2019)

\begin{abstract}
Devising magnetic nanostructures with spatially heterogeneous Dzyaloshinskii-Moriya interaction (DMI) is a promising pathway toward advanced confinement and control of magnetic skyrmions in potential devices. Here we discuss theoretically how a skyrmion interacts with a heterochiral interface using micromagnetic simulations and analytic arguments. We show that a heterochiral interface deflects the trajectory of ferromagnetic (FM) skyrmions, and that the extent of such deflection is tuned by the applied spin-polarized current and the difference in DMI across the interface. Further, we show that this deflection is characteristic of the FM skyrmion, and it is completely absent in the antiferromagnetic (AFM) case. In turn, we reveal that the AFM skyrmion achieves much higher velocities than its FM counterpart, yet experiences far stronger confinement in nanoengineered heterochiral tracks, which reinforces AFM skyrmions as a favorable choice for skyrmion-based devices.
\end{abstract}

DOI: 10.1103/PhysRevB.99.104409

\section{INTRODUCTION}

The interfacially induced Dzyaloshinskii-Moriya interaction (DMI) is a chiral interaction observed in ferromagnetic thin films, e.g., a Co layer, when coupled to nonmagnetic layers with a strong spin-orbit coupling, e.g., the heavy metal Pt [1-3]. The interfacially induced DMI favors the rotation of magnetization at short length scales, giving rise to chiral spin structures of Néel-type, such as cycloids and magnetic skyrmions [4-7]. In particular, magnetic skyrmions are promising candidates for technological applications, such as spin-based information processing and computing devices [7-10]. Most recently, the suggestion of skyrmions in antiferromagnetic (AFM) systems has also increased expectations regarding skyrmion-based devices, since in those systems the skyrmions are not sensitive to stray fields, they move straight along the direction imposed by the applied current, and they present better mobility with lower energy costs [11-17].

The confinement of ferromagnetic (FM) skyrmions in mesoscopic chiral films and tracks has already been thoroughly studied in recent years [18-21]. In the latest development, spatial engineering of DMI has been suggested as an alternative manner of skyrmion guidance and manipulation. Such heterochiral samples have been demonstrated to strongly confine magnetic skyrmions [22], pin them or manipulate their size [23], and increase their lifetime [24] in regions where the DMI is higher. The interest in these results is reinforced by the fact that heterochiral structures can indeed be fabricated experimentally, via engineering of the substrate and/or the capping layer of thin ferromagnetic film $[25,26]$. Bearing in mind the potential of heterochiral systems for the development of skyrmion-based devices, the last piece of the puzzle is to understand skyrmion dynamics in such samples. However, the

\footnotetext{
*milorad.milosevic@uantwerpen.be
}

dynamics of a single magnetic skyrmion while, e.g., crossing the regions with different DMI strengths remains mostly unexplored, while the confinement effects in AFM heterochiral films have not been studied at all to date.

Therefore, in this work we address theoretically the dynamics of FM and AFM skyrmions in heterochiral films, and particularly their interaction with a heterochiral interface (where DMI changes; see, e.g., a suggested realization in Fig. 1). The DMI strength can be modified along the sample using, e.g., lithographic techniques to correspondingly pattern the heavy metal (HM) layer(s) and thereby adjust the interfacially induced DMI $[22,25,26]$. Note that by altering the HM configuration and/or thickness, one might also induce changes in other material parameters, such as the magnetic anisotropy. In this paper, we focus exclusively on the effects of the spatially varied DMI, and therefore we consider the other material parameters homogeneous throughout the sample to avoid misinterpretations of the results. We proceed by employing micromagnetic simulations to show that local canting of the magnetization at the heterochiral interface [22] can be seen as an imposed potential barrier in the Thiele formalism for the center-of-mass of the skyrmion, which causes a characteristic deflection in the trajectory of the FM skyrmion when crossing the heterochiral interface. After verifying it in full micromagnetic simulations, we show that such deflection is completely absent in an analogous antiferromagnetic sample, and that the AFM skyrmion (i) moves much faster than the FM skyrmion, as already predicted in the literature [12], but (ii) experiences far stronger confinement in heterochiral films, so that the critical current needed to push it over a heterochiral interface is much larger than in the FM case. These results promote antiferromagnetic heterochiral films as an advanced platform for skyrmion-based devices.

The paper is organized as follows. In Sec. II we describe the micromagnetic model of ferromagnetic films with interfacially induced DMI and applied spin-polarized current, 


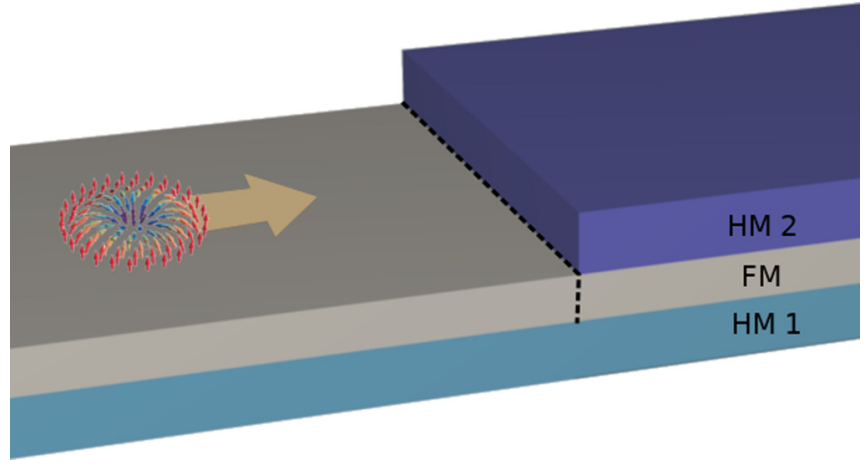

FIG. 1. Schematic representation of an experimental analog of the considered system, a ferromagnetic layer between two HM layers, with a suitably patterned top layer. In the depicted sample, the heterochiral interface is created at the lateral end of the top layer. The dashed line indicates the interface where the DMI changes.

before providing some analytic considerations and the Thiele formalism for the center-of-mass motion of the FM skyrmion driven by in-plane and out-of-plane spin-polarized currents. In Sec. III, we report the characteristic features of skyrmion motion when crossing an interface where DMI changes, for both the ferromagnetic (Sec. III A) and antiferromagnetic (Sec. III B) cases, and we analyze them using both micromagnetic simulations and the Thiele derivations. In Sec. III A 3 we show a possible application of heterochiral interfaces for the manipulation of a skyrmion chain in a nanoengineered circuit. Finally, our results are summarized in Sec. IV.

\section{THEORETICAL FORMALISM}

\section{A. Micromagnetic model}

For the micromagnetic simulations, we employ the micromagnetic simulation package MUMAX $^{3}$ [27] on an ultrathin ferromagnetic film with perpendicular magnetic anisotropy and with spatially inhomogeneous DMI. The local free energy density $\mathcal{E}$, related to the magnetization $\mathbf{M}(x, y)=$ $M_{\mathrm{s}} \mathbf{m}(x, y)$, where $M_{\mathrm{s}}$ is the saturation magnetization and $|\mathbf{m}|=1$, has multiple sources, and we consider the following: exchange, anisotropy, DMI, and demagnetization. We approximate the demagnetization energy by using an effective anisotropy $K_{\text {eff }}=K-\frac{1}{2} \mu_{0} M_{\mathrm{s}}^{2}$, with $K$ the perpendicular magnetic anisotropy and $\mu_{0}$ the vacuum permeability. This approximation is justified by the fact that we are interested in ultrathin films, where dipolar coupling becomes local in the zero-thickness limit [28]. In this work, we do not consider the effects of an external magnetic field, and the Zeeman term of the energy density is therefore zero. The expressions for the remaining energy-density terms are

$$
\begin{aligned}
\mathcal{E}_{\mathrm{ex}} & =A\left[\left(\partial_{x} \mathbf{m}\right)^{2}+\left(\partial_{y} \mathbf{m}\right)^{2}\right], \\
\mathcal{E}_{\text {anis }} & =K_{\text {eff }}\left(1-m_{z}^{2}\right), \\
\mathcal{E}_{\text {DMI }} & =-D\left[m_{x} \partial_{x} m_{z}-m_{z} \partial_{x} m_{x}+m_{y} \partial_{y} m_{z}-m_{z} \partial_{y} m_{y}\right] .
\end{aligned}
$$

For the simulations of the ferromagnetic case, we consider the following parameters: saturation magnetization:
$M_{\mathrm{s}}=580 \mathrm{kA} \mathrm{m}^{-1}$, exchange stiffness: $A=15 \mathrm{pJ} \mathrm{m}^{-1}$, and perpendicular anisotropy: $K=0.8 \mathrm{MJ} \mathrm{m}^{-3}\left(K_{\text {eff }}=\right.$ $0.6 \mathrm{MJ} \mathrm{m}^{-3}$ ), stemming from the experimental results on $\mathrm{Co} / \mathrm{Pt}$ systems [29,30]. The used values of the DMI constant, $D$, will be specified in the sections below. For all simulations, we consider a system discretized into cells of size $1 \times 1 \times$ $0.4 \mathrm{~nm}^{3}$. The dynamics of the magnetization is governed by the Landau-Lifshitz-Gilbert (LLG) equation

$$
\frac{d \mathbf{m}}{d t}=\frac{\gamma}{1+\alpha^{2}}\left\{\mathbf{m} \times \mathbf{H}_{\mathrm{eff}}+\alpha\left[\mathbf{m} \times\left(\mathbf{m} \times \mathbf{H}_{\mathrm{eff}}\right)\right]\right\},
$$

where $\gamma$ is the gyromagnetic ratio and $\alpha$ is the damping factor. $\mathbf{H}_{\text {eff }}$ is the effective magnetic field given by the functional derivative of the free energy $E=\int\left(\mathcal{E}_{\text {ex }}+\mathcal{E}_{\text {anis }}+\mathcal{E}_{\text {dmi }}\right) d V$ with respect to the magnetization: $\mathbf{H}_{\mathrm{eff}}=-\frac{1}{\mu_{0} M_{\mathrm{s}}} \delta E / \delta \mathbf{m}$.

The Néel skyrmion in a chiral magnetic film can be driven by two different scenarios [31]: (i) by an in-plane spinpolarized current (CIP) applied into the ferromagnetic layer, or (ii) by an electrical current applied into the HM layer, which due to the spin Hall effect gives rise to a spin-polarized current perpendicular to the plane (CPP) [15,31-33]. In this work, we explore both scenarios. For simulations of the spin-transfer torque (STT) associated with the CIP scenario, the Zhang and Li STT term [34] $\boldsymbol{\tau}_{\mathrm{ZL}}=\frac{b}{1+\alpha^{2}}\{\mathbf{m} \times[\mathbf{m} \times(\mathbf{j}$. $\nabla) \mathbf{m}]+(\beta-\alpha) \mathbf{m} \times(\mathbf{j} \cdot \nabla) \mathbf{m}\}$ was added to the LLG equation, where $\mathbf{j}$ is the current density, $\beta$ is the nonadiabatic factor, and $b=P \mu_{B} / e M_{s}\left(1+\beta^{2}\right)$, with $P$ the polarization of the current density, $\mu_{B}$ the Bohr magneton, and $e$ the electron charge. In the CPP scenario, the electrical current applied into the HM layer results in a spin current injected into the FM along the $z$ direction, with $\mathbf{m}_{p}=-\operatorname{sgn} \theta_{\mathrm{SH}}\left(\hat{z} \times \hat{j}_{\mathrm{hm}}\right)$ the orientation of the injected spins $[15,31,35,36]$, where $\theta_{\mathrm{SH}}$ is the spin-Hall angle of the HM. In this work we assume $\theta_{\mathrm{SH}}$ positive, which, for instance, is the case in a Pt layer [37] $\mathbf{j}_{\mathrm{hm}}$ is the current density flowing through the heavy-metal layer. In MUMAX ${ }^{3}$ one can simulate a similar scenario by considering a fixed layer, with polarization vector $\mathbf{m}_{p}$, on top of the film and the applied current injected along the $\hat{z}$ direction. In this situation, the Slonczewski STT term $[38,39]$ $\boldsymbol{\tau}_{\mathrm{SL}}=\frac{1}{1+\alpha^{2}} \frac{j_{z} \hbar P}{2 e M_{s} d}\left[\mathbf{m} \times\left(\mathbf{m}_{p} \times \mathbf{m}\right)+\alpha \mathbf{m} \times \mathbf{m}_{p}\right]$ was added to the LLG equation, with $d$ the thickness of the ferromagnetic layer and $j_{z}=\theta_{\mathrm{SH}} j_{\mathrm{hm}}$ the spin current density induced along the $z$ direction. For both the CIP and the CPP scenarios, the polarization rate of the spin-polarized current was fixed at $P=0.4$.

For the antiferromagnetic samples, we consider the same parameters of the FM case, except for the negative exchange stiffness $A=-15 \mathrm{pJ} \mathrm{m}^{-1}$. Note that MUMAX ${ }^{3}$ was originally developed for simulations of FM systems in the continuous field approximation. However, once we consider the AFM system, which comprises two sublattices of reversely aligned spins, we end up performing an atomistic simulation (albeit with a large lattice parameter), where the finite-differences derivatives performed by MUMAX ${ }^{3}$ are mathematically equivalent to the classical Heisenberg model (see, e.g., Appendix A) The STT can be applied also to the AFM system provided one considers an ultrasmall mesh size in the micromagnetic simulations [13-15,40,41]. In this work, we simulate only the CPP-driven AFM skyrmion. Note that one cannot 
straightforwardly use the CIP scenario in the micromagnetic simulations since spatial derivatives are involved in the STT term and the reversely aligned magnetization of the AFM system can no longer be described by a differentiable field.

\section{B. Thiele equation for skyrmion dynamics}

The Thiele equation describes the dynamics of the center of mass of the skyrmion by assuming a rigid body motion of the spin texture [31-33,42], and it can be written out for both CIP and CPP scenarios.

The Thiele equation for the CIP scenario reads

$$
\mathbf{G} \times(\boldsymbol{v}-\dot{\mathbf{r}})+\mathcal{D}(\beta \boldsymbol{v}-\alpha \dot{\mathbf{r}})-\nabla V(\mathbf{r})=0,
$$

where $\mathbf{G}=\mathcal{G} \hat{z}=4 \pi Q \hat{z}$ is the gyromagnetic coupling vector, with $Q$ the skyrmion number; $\dot{\mathbf{r}}=\dot{x} \hat{x}+\dot{y} \hat{y}$ is the drift velocity; $\boldsymbol{v}$ is the velocity of the conduction electrons associated with the spin-polarized current; $V$ is the potential stemming from an external force, boundaries, or impurities; and $\mathcal{D}$ represents the dissipative tensor, with components $\mathcal{D}_{i j}=\int d^{2} r \partial_{i} \mathbf{m}$. $\partial_{j} \mathbf{m}=\mathcal{D} \delta_{i j}$ (for the range of parameters considered in this work, $\mathcal{D} \approx 4 \pi-8 \pi$, see Appendix B). If one considers a current applied along the $x$ direction, i.e., $v_{y}=0$, then the Thiele equation can be separated into its two components, which for the case of $V=0$ yields

$$
\begin{aligned}
& \dot{x}=\left(\frac{\mathcal{G}^{2}+\mathcal{D}^{2} \alpha \beta}{\mathcal{G}^{2}+\mathcal{D}^{2} \alpha^{2}}\right) v_{x}, \\
& \dot{y}=\left(\mathcal{G D} \frac{\alpha-\beta}{\mathcal{G}^{2}+\alpha^{2} \mathcal{D}^{2}}\right) v_{x} .
\end{aligned}
$$

The above equations describe the skyrmion velocity due to the applied current in the absence of external forces and impurities, where the skyrmion velocity is constant for a fixed applied current. Notice that the skyrmion undergoes a transverse motion, $\dot{y} \neq 0$ (when $\alpha$ differs from $\beta$ ), because it carries a nonzero skyrmion number $(\mathcal{G} \neq 0)$. If we consider $V(\mathbf{r})=V(x)$, the Thiele equation leads to

$$
\begin{gathered}
\dot{y}=\frac{\mathcal{G}}{\mathcal{D} \alpha}\left(v_{x}-\dot{x}\right), \\
\left(\frac{\mathcal{G}^{2}}{\mathcal{D} \alpha}+\mathcal{D} \beta\right) v_{x}-\left(\frac{\mathcal{G}^{2}}{\mathcal{D} \alpha}+\mathcal{D} \alpha\right) \dot{x}=\frac{d V}{d x} .
\end{gathered}
$$

Note that the $x$ component of the skyrmion velocity is directly affected by the external potential, and, consequently, the Magnus force (represented by the $\mathcal{G}$ term), which drives the skyrmion along the $y$ direction, is also affected. Indeed, taking the variation $\delta \dot{y} \equiv \dot{y}(t+d t)-\dot{y}(t)$ of Eq. (5a), for a fixed current density, we obtain

$$
\delta \dot{y}=-\frac{\mathcal{G}}{\mathcal{D} \alpha} \delta \dot{x},
$$

which means that, if the external potential is attractive $(d V / d x<0)$ or repulsive $(d V / d x>0)$, the skyrmion trajectory is deflected to the $-\mathcal{G} \hat{y}$ or $+\mathcal{G} \hat{y}$ direction, respectively, depending on the skyrmion number.

In the case of a repulsive external potential, the critical current for the skyrmion to overcome such an energy barrier is given by choosing $\dot{x}=0$ for the maximal value of $F=d V / d x$ in Eq. (5b), i.e.,

$$
v_{x}^{c}=\frac{F_{\max }}{\frac{\mathcal{G}^{2}}{\mathcal{D} \alpha}+\mathcal{D} \beta} .
$$

For the limit of small $\alpha$ and $\beta(\alpha \sim \beta \ll 1)$, the critical current can be approximated as $v_{x}^{c}=\frac{F_{\max } \mathcal{D} \alpha}{\mathcal{G}^{2}}$. In the same way, Eq. (5b) results in $\left(v_{x}-\dot{x}\right) \approx \frac{\mathcal{D} \alpha}{\mathcal{G}^{2}} \frac{d V}{d x}$. Substituting this expression into Eq. (5a), we obtain the velocity of the skyrmion in the $y$ direction:

$$
\dot{y} \approx \frac{1}{\mathcal{G}} \frac{d V}{d x},
$$

which depends only on the external potential $V$. The maximal velocity can be written as $\dot{y}_{\max }=F_{\max } / \mathcal{G}$.

Similar results are obtained for a Néel skyrmion driven by the CPP scenario. In this case, the skyrmion motion is described by the modified Thiele equation [31-33]:

$$
-\mathbf{G} \times \dot{\mathbf{r}}-\alpha \mathcal{D} \dot{\mathbf{r}}+4 \pi \mathcal{B} \mathbf{j}_{\mathrm{hm}}-\nabla V(\mathbf{r})=0,
$$

where $\mathbf{j}_{\mathrm{hm}}$ is the current density flowing through the heavy metal, which gives rise to a spin-polarized current perpendicular to the plane. The parameter $\mathcal{B}$ quantifies the efficiency of the spin-Hall effect. Now we consider $\mathbf{j}_{\mathrm{hm}}=j_{\mathrm{hm}} \hat{y}$. For the case of $V=0$, Eq. (9) yields

$$
\begin{aligned}
& \dot{x}=\frac{\mathcal{G}}{\mathcal{G}^{2}+\alpha^{2} \mathcal{D}^{2}} 4 \pi \mathcal{B} j_{\mathrm{hm}}, \\
& \dot{y}=\frac{\alpha \mathcal{D}}{\mathcal{G}^{2}+\alpha^{2} \mathcal{D}^{2}} 4 \pi \mathcal{B} j_{\mathrm{hm}} .
\end{aligned}
$$

Note that, for $\alpha \ll 1$, the Magnus term dominates $\dot{x} \gg \dot{y}$, and the relevant motion is along the $x$ direction. If we consider $V(\mathbf{r})=V(x)$, the modified Thiele equation leads to

$$
\begin{array}{r}
\dot{y}=\frac{1}{\mathcal{D} \alpha}\left(4 \pi \mathcal{B} j_{\mathrm{hm}}-\mathcal{G} \dot{x}\right), \\
-\left(\frac{\mathcal{G}^{2}}{\mathcal{D} \alpha}+\mathcal{D} \alpha\right) \dot{x}+\frac{4 \pi \mathcal{B} \mathcal{G}}{\mathcal{D} \alpha} j_{\mathrm{hm}}=\frac{d V}{d x} .
\end{array}
$$

Taking the variation $\delta \dot{y}$ of Eq. (11a) for a fixed current density, we recover Eq. (6). Therefore, the presence of an external potential deflects the skyrmion trajectory in the same direction as in the CIP scenario. In the same way, the critical current for the skyrmion to overcome a repulsive potential is obtained by choosing $\dot{x}=0$ in Eq. (11b), i.e.,

$$
4 \pi \mathcal{B} j_{\mathrm{hm}}^{c}=\frac{\mathcal{D} \alpha}{\mathcal{G}} F_{\max } .
$$

Finally, for the limit of $\alpha \ll 1$, Eq. (11b) becomes $\left(4 \pi \mathcal{B} j_{\mathrm{hm}}-\right.$ $\mathcal{G} \dot{x}) \approx \frac{\mathcal{D} \alpha}{\mathcal{G}} \frac{d V}{d x}$. By substituting this expression into Eq. (11a), we recover Eq. (8) for the skyrmion velocity in the $y$ direction.

\section{RESULTS AND DISCUSSION}

\section{A. Ferromagnetic skyrmion}

\section{Skyrmion trajectory when facing nonuniform canting of the background magnetization}

In this work, we are interested in the skyrmion motion in heterochiral systems, particularly the behavior of the skyrmion trajectory while crossing an interface where DMI 

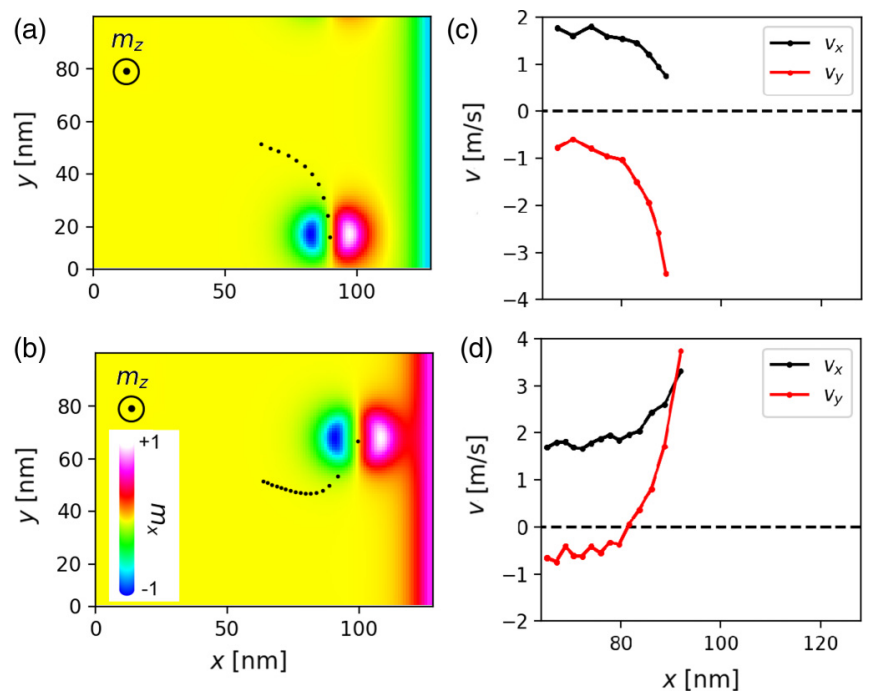

FIG. 2. Skyrmion trajectories (trail of black dots) in the presence of (a) negative and (b) positive canting of the background magnetization at the right edge of the sample. (c) Center-of-mass velocities of the skyrmion in plot (a). (d) Center-of-mass velocities of the skyrmion in plot (b). Here $j=5 \times 10^{10} \mathrm{~A} \mathrm{~m}^{-2}, \alpha=0.3$, and $\beta=0$. The center of mass is calculated as the mean point of the region where $m_{z}=0$.

changes. As the simplest case of a heterochiral film, we consider a system where the DMI strength, $D$, varies only in the $x$ direction as $D=D_{1}$ for $x<x_{0}$, and $D=D_{2}$ for $x>x_{0}$, with $D_{1}$ and $D_{2}$ constant. For this geometry, it was shown in Ref. [22] that canting of the magnetization is induced at the interface $x=x_{0}$, and that the magnetization profile is given by

$$
\theta(x)=2 \arctan \left(e^{-\left|\left(x-x_{0}\right) / \xi\right|} \tan \frac{\theta_{0}}{2}\right),
$$

where $\theta(x)$ is the angle of the spins with respect to the $z$ axis, $m=(\sin \theta, 0, \cos \theta), \xi=\sqrt{A / K_{\text {eff }}}$, and

$$
\theta_{0}=\arcsin \frac{D_{2}-D_{1}}{\pi D_{c}}
$$

is the canting angle at the interface, with $D_{c}=4 \sqrt{A K_{\mathrm{eff}}} / \pi$. Notice that in Ref. [22] the DMI parameter takes opposite sign to the one used here. The canting of the magnetization at the interface can be either positive or negative, depending on the difference between the DMI strengths $D_{1}$ and $D_{2}$.

It now becomes of interest to first understand what happens to the skyrmion trajectory when encountering such nonuniform canting of the background magnetization. In Fig. 2 we show the result of a simulation performed in the micromagnetic framework for the skyrmion trajectory in a thin ferromagnetic film with uniform DMI, here fixed at $D=$ $0.8 D_{c}$, and artificially imposed canting of spins on the right sample boundary. We consider a sample of size $128 \times 96 \times$ $0.4 \mathrm{~nm}^{3}$, with periodic boundary conditions in the $y$ direction. The skyrmion is initialized in the center of the simulated region, and the energy is minimized numerically. An in-plane polarized current is then applied in the $-\hat{x}$ direction (CIP scenario). The skyrmion undergoes a transverse motion due
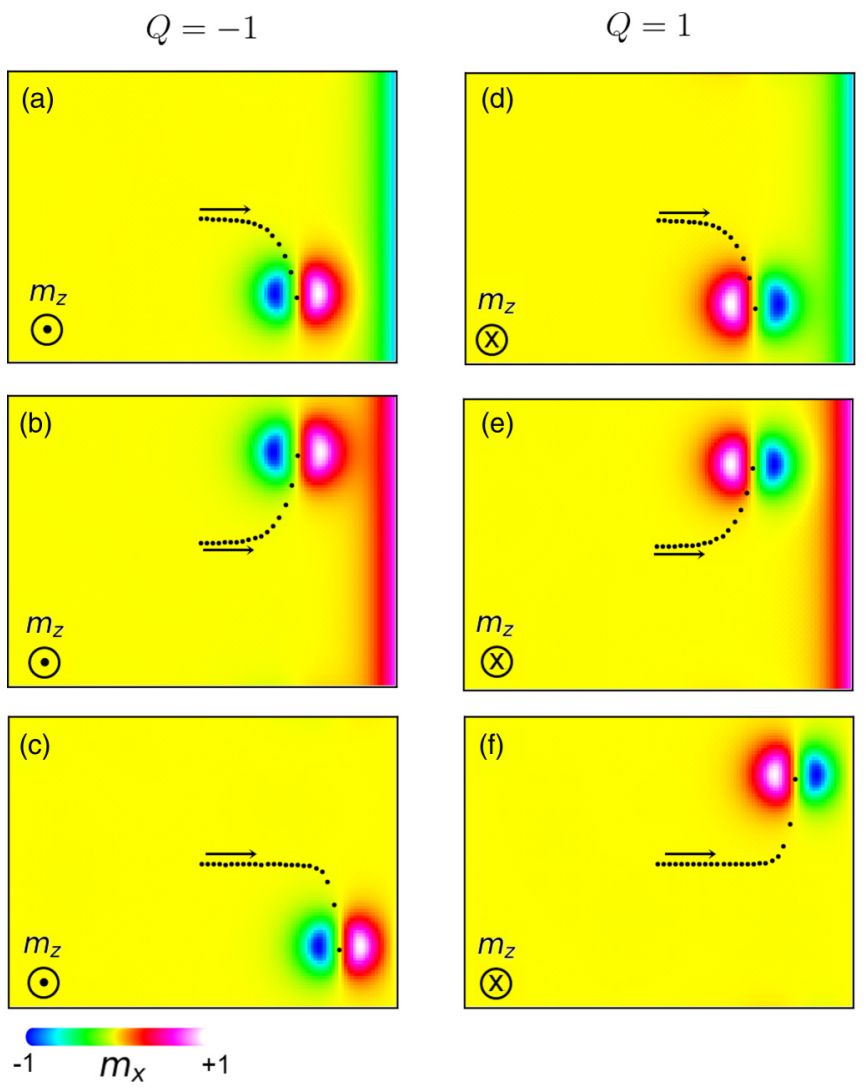

FIG. 3. Skyrmion trajectories (given as a trail of black dots) for $Q=-1$ (a)-(c) and $Q=1$ (d)-(f), for different canting of the magnetization at the edge, $m_{\text {fixed }}^{x}=-\sin \theta_{\text {edge }},+\sin \theta_{\text {edge }}$, and 0 , respectively, from top to bottom, with $\theta_{\text {edge }}=\frac{\pi}{4}$. The applied current density is $j=5 \times 10^{10} \mathrm{~A} \mathrm{~m}^{-2}$.

to the Magnus force. To address the effect of nonuniform canting of the magnetization (as expected at a heterochiral interface), we fix a column of spins at the right side of the sample as $\mathbf{m}_{\text {fixed }}=\left(-\sin \theta_{\text {edge }}, 0, \cos \theta_{\text {edge }}\right)$ in Fig. 2(a), and $\mathbf{m}_{\text {fixed }}=\left(\sin \theta_{\text {edge }}, 0, \cos \theta_{\text {edge }}\right)$ in Fig. 2(b), with canting angle $\theta_{\text {edge }}=\frac{\pi}{4}$. The fixed columns spread the canting of the magnetization in the vicinity of the edge, which then affects the skyrmion trajectory. Although these examples are not ideally realistic, they will be useful to understanding the results of the next section. Figures 2(c) and 2(d) shows that the induced canting can be seen as either repulsive (c) or attractive (d) external potential for the skyrmion. In those two cases, the Magnus force pushes the skyrmion in different directions $(-\hat{y}$ and $+\hat{y})$. These results are in accordance with Eq. (6) if one considers the local canting of the magnetization as an external potential for the center of mass of the skyrmion, which is a reasonable assumption, since the energy cost for the skyrmion to flip the background spins during its motion is higher if the background spins are in the opposite direction [Fig. 2(a)] than if those have the same polarity as the skyrmion [Fig. 2(b)]. We have obtained analogous results for the CPP scenario.

To provide a better comparison of the simulations with the analytic results, we next consider the case $\alpha \ll 1$ and $\beta=0$. In this case, the relevant motion in the $y$ direction will be given solely by the effect of the external potential 


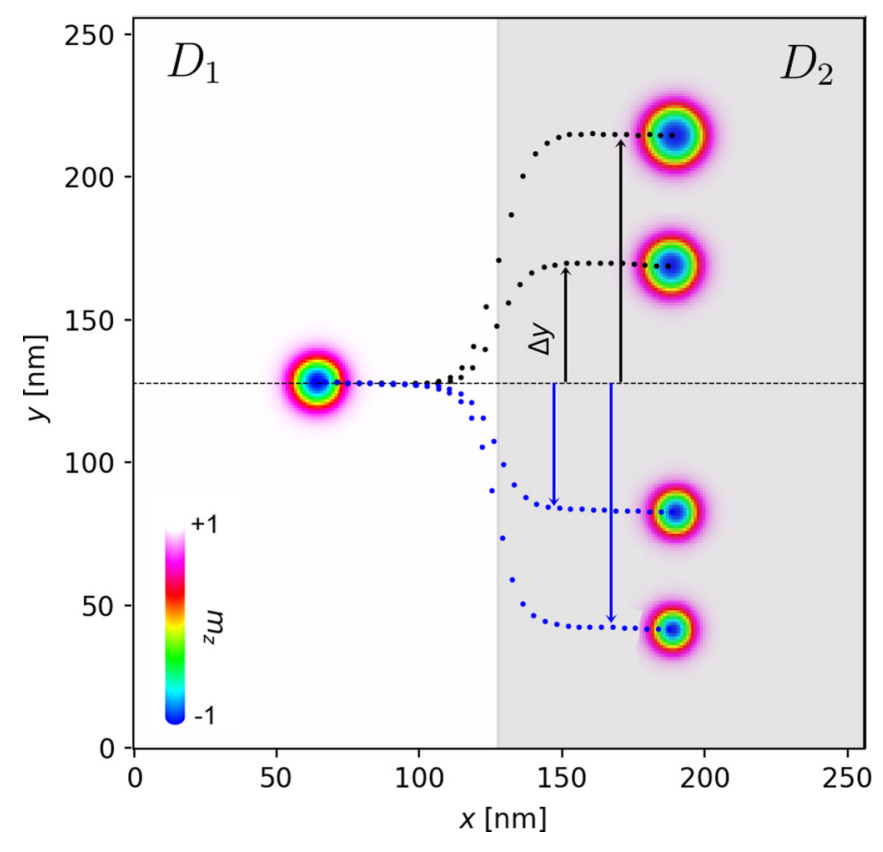

FIG. 4. The skyrmion is initialized on the left side of the diagram. Depending on the difference of the DMI strengths, $D_{1}$ and $D_{2}$, the skyrmion deflection is positive $(\Delta y>0$, black arrows) or negative ( $\Delta y<0$, blue arrows). The dots show the respective trajectories of the skyrmion for $j=2 \times 10^{11} \mathrm{~A} \mathrm{~m}^{-2}$ and $\Delta D / D_{c}=0.05,0.025$, -0.025 , and -0.05 , respectively, top to bottom.

[see Eq. (8)]. In Fig. 3, we take $\alpha=0.02$ (within the typical range $\alpha \sim 10^{-3}-10^{-2}$ for skyrmion-hosting materials [31,43-46]), and we perform the same simulation of Fig. 2, but now for six different situations: for the skyrmion numbers $Q= \pm 1$, and the fixed magnetizations at the right edge $m_{\text {fixed }}^{x}=-\sin \theta_{\text {edge }},+\sin \theta_{\text {edge }}$ and 0 , with $\theta_{\text {edge }}=\frac{\pi}{4}$. In the last case, the fixed spins do not induce any canting of the magnetization, but they increase the necessary energy to flip their neighbors and must act as a repulsive potential for both considered skyrmion numbers. With such examples, we look for the corroboration of Eq. (6) for predicted deflection of the skyrmion trajectory. Comparing Figs. 3(a) and 3(b) to Figs. 3(d) and 3(e), we change the skyrmion number [consequently the sign of $\mathcal{G}$ in Eq. (6) as well], but we also change the canting effect from repulsive to attractive and vice versa. Therefore, the skyrmion is deflected in the same direction for both $Q= \pm 1$. Comparing Fig. 3(c) to Fig. 3(f), the skyrmion number changes, but the fixed spins act as a repulsive barrier in both situations, hence the skyrmion is deflected in opposite directions for opposite topological charge. These results are in complete accordance with Eq. (6) and will be useful to understanding the results of the next sections. For the CPP scenario, we obtained similar results when choosing $\alpha=0.02$.

\section{Skyrmion trajectory while crossing an interface where DMI changes}

The examples of the previous subsection are not realistic, however, as we saw before, similar canting of the magnetization is intrinsic to the DMI interface(s) in a heterochiral ferromagnetic film. Therefore, we study next the trajectory

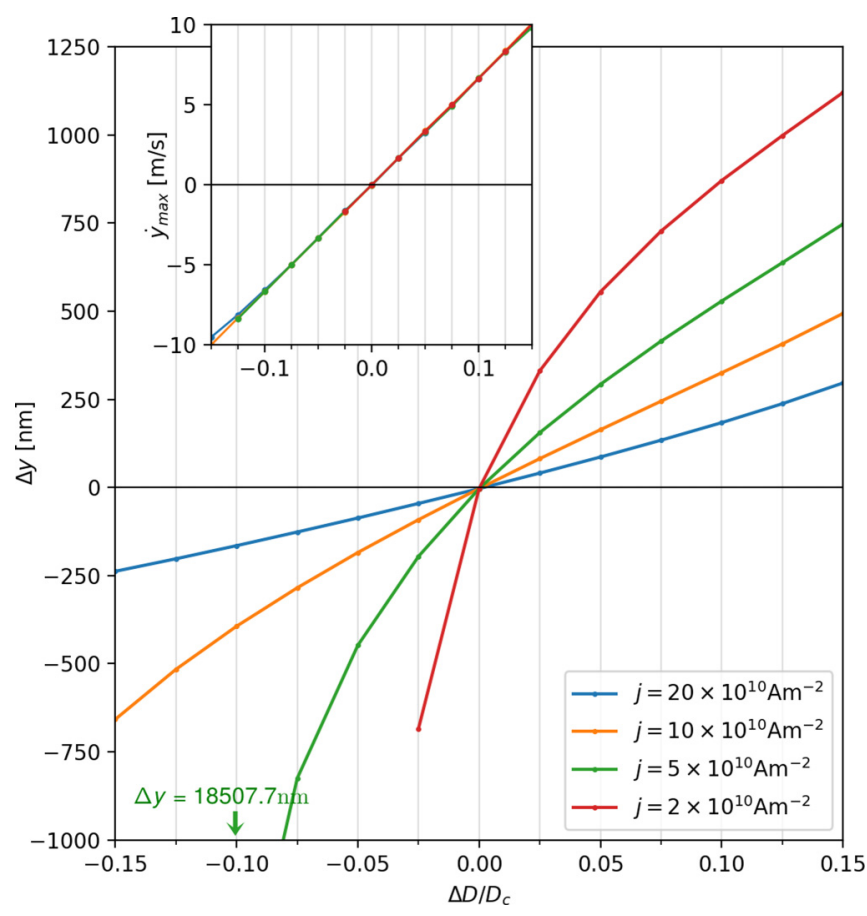

FIG. 5. Main panel exhibits the deflection in the skyrmion trajectory, $\Delta y$, for different values of applied current $j$ and the change in DMI across the interface $\Delta D=D_{2}-D_{1}$, with $D_{1}=0.8 D_{c}$ fixed. The inset shows the maximal velocity of the skyrmion in the $y$ direction (along the interface) as a function of $\Delta D$ for different values of $j$.

of a single skyrmion while crossing an interface where DMI changes. In the simulations, we consider a sample of size $256 \times 256 \times 0.4 \mathrm{~nm}^{3}$, with DMI strength $D_{1}$ for $x<x_{0}$ and $D_{2}$ for $x>x_{0}$, where $x_{0}=128 \mathrm{~nm}$. The skyrmion is initialized at the position $x=64 \mathrm{~nm}, y=128 \mathrm{~nm}$ (see Fig. 4), and we consider periodic boundary conditions in the $y$ direction. An in-plane current is applied along $-\hat{x}$ (CIP scenario), with $\alpha=0.02$ and $\beta=0$, such that the relevant motion in the $y$ direction will be given solely by the effect of the heterochiral interface (the effects of the nonadiabatic parameter, $\beta$, to the skyrmion trajectory are shown in Appendix C). As expected from the previous discussion, the skyrmion is deflected at the interface along $\pm \hat{y}$, depending on the canting direction, which in turn depends on the DMI strengths $D_{1}$ and $D_{2}$.

To illustrate the role of different parameters, we calculate the skyrmion deflection $\Delta y$ after the skyrmion reaches the position $x=192 \mathrm{~nm}$ (as shown in Fig. 4) for selected values of DMI strengths and applied currents. Figure 5 shows the skyrmion deflection after crossing the interface as a function of $\Delta D=D_{2}-D_{1}$, with $D_{1}=0.8 D_{c}$ fixed. For high currents, the deflections are smaller than those observed for low currents, and antisymmetric for $\Delta D$ positive or negative, since the energy barrier induced by the interface is small when compared to the kinetic energy induced by the applied current. On the other hand, for low currents, the skyrmion motion can be completely blocked by the repulsive potential induced by $\Delta D<0$ if $j<j_{c}(\Delta D)$, where $j_{c}$ is the critical current for the skyrmion to overcome the interface. The more negative $\Delta D$ is, the higher is the necessary current for the skyrmion to 
overcome the interface. For example, for $j=2 \times 10^{10} \mathrm{~A} \mathrm{~m}^{-2}$ in Fig. 5, the skyrmion cannot cross the interface for $\Delta D \leqslant$ $-0.05 D_{c}$, and it continues the motion purely in the $y$ direction, along the interface. Notice that for the considered parameters, the $y$ component of the skyrmion velocity does not depend on the applied current [see Eq. (8)]. The maximal velocity of the skyrmion in the $y$ direction, for a fixed $\Delta D$, is the same for all current values, as confirmed by the graph in the inset of Fig. 5. However, for low currents the skyrmion takes a longer time to cross the interface, which translates into a larger deflection. The largest deflections are observed when the applied current is just above $j_{c}$, e.g., for the case of $j=$ $5 \times 10^{10} \mathrm{~A} \mathrm{~m}^{-2}$ in Fig. 5, where the skyrmion overcomes the interface with $\Delta D=-0.1 D_{c}$ after a rather extreme deflection of $\Delta y=-18.5 \mu \mathrm{m}$.

Comparing the graph in the inset of Fig. 5, where $\dot{y}_{\max }$ varies linearly with $\Delta D$, with Eqs. (8) and (14), we obtain

$$
F_{\max } \approx c \mathcal{G} \Delta D=c \pi D_{c} \mathcal{G} \sin \theta_{0},
$$

where $c$ is the slope of $\dot{y}_{\max }(\Delta D)$ characteristic in the inset of Fig. 5, and $\theta_{0}$ is the canting angle at the interface (from the graph, we obtained $c \approx 67 / D_{c} \mathrm{~ms}^{-1}$ ). Note that, since the external potential $V$ does not depend on the applied current scenario and Eq. (8) is valid for both CIP and CPP scenarios, the graph in the inset of Fig. 5 and consequently Eq. (15) are general results for a ferromagnetic skyrmion. Therefore, the critical current, given by Eqs. (7) and (12), depends linearly on $\Delta D$ for both CIP and CPP scenarios. Note that the dissipative factor $\mathcal{D}$ in Eqs. (7) and (12) depends on the skyrmion size, which in turn depends on the material parameters, e.g., the DMI strength. However, as will be shown in Fig. 9 of the next section, for the considered range of parameters, such linear dependence is preserved in the CPP scenario for different values of $\alpha$.

\section{Multichannel skyrmion bit sequencer}

Based on our findings, the heterochiral interface can be used to very precisely guide the skyrmion motion in a more complex circuitry, for example to selectively "write" skyrmions in one of multiple nanotracks, or to selectively direct a skyrmion to one of the many logical gates in a larger skyrmion microprocessor. We exemplify here such an application of a heterochiral interface for the targeted manipulation of a chain of skyrmions by pulsed current. Although simplistic, this example is intended for the reader to creatively visualize other possible uses of heterochiral systems.

In this example, we consider a rectangular ferromagnetic film of size $880 \times 634 \times 0.4 \mathrm{~nm}^{3}$, where high-DMI tracks are engineered (by a suitable heavy-metal capping layer; see Fig. 6) with DMI strengths of $D_{1}=0.8 D_{c}$ (single track on the left) and $D_{2}=0.75 D_{c}$ (six tracks on the right side). A skyrmion chain, containing skyrmions labeled Sk1, Sk2, and Sk3 and separated by $115 \mathrm{~nm}$, is initialized in the $D_{1}$ track on the left side of the sample. A current pulse is then applied along the $-\hat{x}$ direction (CIP scenario), which induces motion of skyrmions along the $+\hat{x}$ direction. The duration and intensity of subsequent current pulses is designed in such a manner that each skyrmion reaches the heterochiral interface

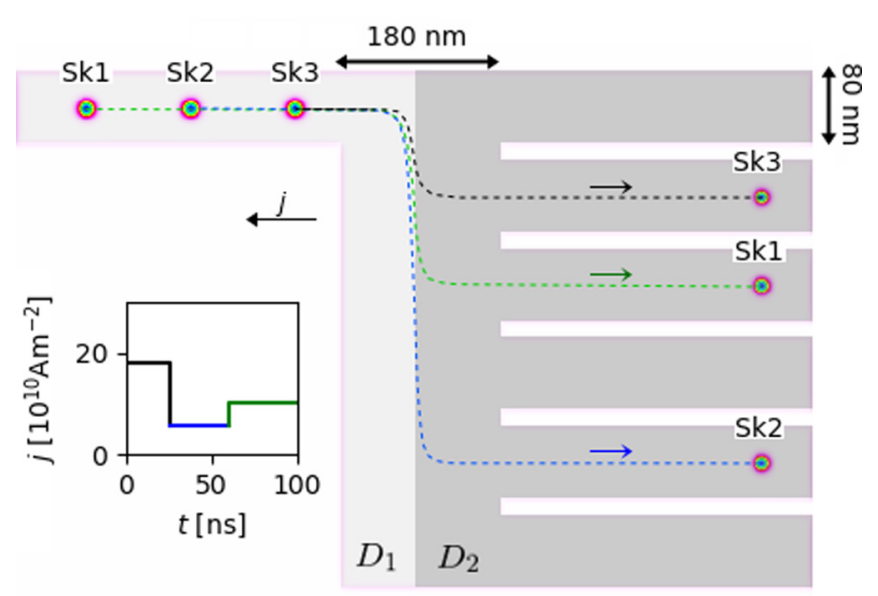

FIG. 6. Selective deflection of a skyrmion chain into multiple nanotracks, using the properties of a heterochiral interface. The dashed lines indicate the trajectory of each skyrmion during the simulation, for a series of current pulses of $j=18 \times 10^{10} \mathrm{~A} \mathrm{~m}^{-2}$ for $0<t<25 \mathrm{~ns}, j=5.5 \times 10^{10} \mathrm{~A} \mathrm{~m}^{-2}$ for $25<t<60 \mathrm{~ns}$, and $j=10 \times 10^{10} \mathrm{~A} \mathrm{~m}^{-2}$ for $t>60 \mathrm{~ns}$, in a sample with $\alpha=0.02$, $\beta=0, D_{1}=0.8 D_{c}$, and $D_{2}=0.75 D_{c}\left(\Delta D / D_{c}=-0.05\right)$.

under a different current density, and thereby experiences different deflection of its trajectory. Moreover, the intensity of the pulses is chosen according to Fig. 5, so that the deflection of the skyrmions exactly corresponds to the entry point of one of the six tracks on the right side of the sample [47].

Obviously, the exact duration and intensity of the current pulses has to be precisely engineered for a particular realization of the sample, depending on the separation of skyrmions in the initial chain and the values of all relevant parameters including the change of DMI across the interface. Nevertheless, once optimized, such an interface can be very reliably used to write skyrmions in multiple channels in any desired sequence, as we show in the animated data in the supplemental material [47]. We remind the reader that current-induced deflection of a FM skyrmion at a heterochiral interface can easily exceed ten micrometers (as shown in the previous section), hence a large number of nanotracks could be very controllably accessed in this manner. We stress that such controlled manipulation is needed for more complex skyrmion-based computing and storage circuits. For instance, it could be used to selectively place the skyrmions in the input branches of (many) skyrmion-based logic gates [48], or to precisely write information in multibit memory cells.

\section{B. Antiferromagnetic skyrmion}

Antiferromagnetic (AFM) skyrmions are expected to combine the advantages of antiferromagnets with those of skyrmions regarding spintronic applications. AFM skyrmions have zero net topological charge, and simulations of their current-induced motion have shown that accordingly they move straight along the direction imposed by the applied current [12-15]. This is considered advantageous for applications, because as opposed to ferromagnetic skyrmions, their antiferromagnetic counterparts are not driven toward the boundary of the hosting magnetic structures, where 

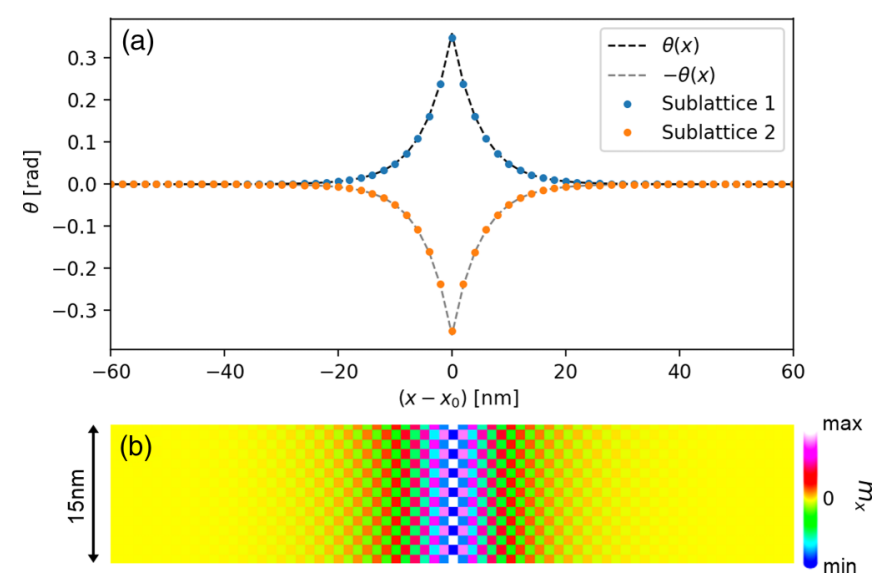

FIG. 7. (a) Canting of the magnetization $\theta(x)$ at a DMI interface of a heterochiral AFM system, plotted separately for each sublattice, for $D_{2}-D_{1}=1.1 D_{c}$. Dashed lines represent the analytic result for the FM system, given by Eq. (13). (b) Snapshot zoom of the configuration obtained after minimizing the energy numerically.

they can collapse. Additional benefits arise from their antiferromagnetic nature, i.e., their insensitivity to parasitic stray fields [12]. In what follows, we address in more detail the behavior of AFM skyrmions in heterochiral samples.

Antiferromagnetic skyrmions have been recently intensively studied regarding their spin structure, their stability, and their motion [12-16,49-51]. The AFM skyrmion comprises a two-sublattice structure, where each sublattice (indexed 1 and 2) contains half of the spins of the system and has the opposite magnetization of the other sublattice. In this way, the topological numbers projected to each sublattice satisfy $Q_{1}=-Q_{2}$. The opposing topological index of two sublattices causes the exact cancellation of the Magnus force in the presence of current, so the antiferromagnetic skyrmion moves along the direction of the current. The velocity of the AFM skyrmion driven by a current density is inversely proportional to the damping factor $\alpha$, and the AFM skyrmion can move much faster than the FM one for weak damping, possibly reaching $\mathrm{km} / \mathrm{s}$ while remaining stable [12-15,17].

To understand the dynamics of the AFM skyrmion while crossing an interface where the DMI changes, we first simulate, in the micromagnetic framework, the AFM ground state in the presence of such an interface. Here, we consider a sample of size $256 \times 100 \times 0.4 \mathrm{~nm}^{3}$ with DMI strength $D_{1}$ for $x<x_{0}$ and $D_{2}$ for $x>x_{0}$, with $x_{0}=128 \mathrm{~nm}$. Figure 7(b) shows a snapshot zoom of the configuration obtained after minimizing the energy numerically. Notice that the induced canting $\left(m_{x}=\sin \theta\right)$ points in opposite directions in each sublattice. As shown in Fig. 7(a), the canting induced at each sublattice follows the analytic result for the FM system (dashed lines), given by Eq. (13).

In the presence of canting induced by the DMI interface, we expect skyrmion scattering at each sublattice to follow the FM result of Fig. 3. We recall that the sublattices have opposite topological charge and induce opposite canting at the interface, hence the effective motion of the AFM skyrmion, given by a combination of the two sublattices, is a combination of either Figs. 3(a) and 3(e), or Figs. 3(b) and 3(d).
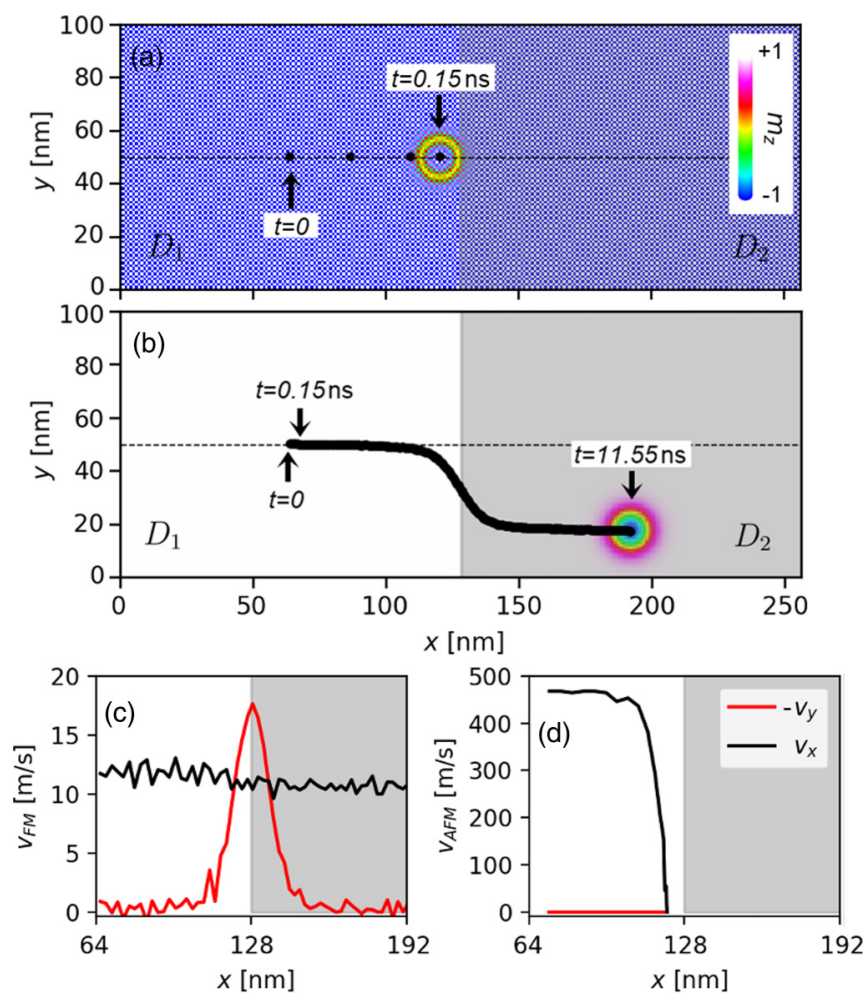

FIG. 8. Snapshots of the calculated spin configurations during simulation for the AFM (a) and FM (b) skyrmion driven by the CPP scenario. The trail of black dots indicates the skyrmion trajectory. (a) The AFM skyrmion reaches the interface after $t=0.15 \mathrm{~ns}$, where its movement is completely blocked. (b) The FM skyrmion moves slower than the AFM one, but it can cross the interface after a sufficiently long time. (c) Center-of-mass velocities of the FM skyrmion, during motion shown in (b). (d) Center-of-mass velocities of the AFM skyrmion, during motion shown in (a). The spin current is applied along the $\hat{z}$ direction but polarized along $+\hat{y}\left(\hat{j}_{\mathrm{hm}}=-\hat{x}\right)$ for the AFM case, and $-\hat{x}\left(\hat{j}_{\mathrm{hm}}=-\hat{y}\right)$ for the FM case, with current density $j=2 \times 10^{10} \mathrm{~A} \mathrm{~m}^{-2}$, DMI strengths $D_{1}=0.8 D_{c}$ and $D_{2}=$ $0.775 D_{c}$, and damping parameter $\alpha=0.02$. The center of mass is calculated as the mean point of the region where $m_{z}=0$.

Therefore, the characteristic deflection observed for the FM skyrmion while crossing the interface is completely absent (canceled out) in the AFM system. However, the attractive or repulsive effect in the $x$ direction is still expected.

The dynamics of the magnetization in the micromagnetic simulations is controlled by applying a spin current perpendicular to the plane (CPP scenario). Since the DMI interface is always either attractive in both sublattices [combination of Figs. 3(b) and 3(d)] or repulsive in both sublattices [combination of Figs. 3(a) and 3(e)], the DMI interface can be seen as an external potential in the modified Thiele equation [Eq. (9)], for a single lattice with $G=0$. Since the AFM skyrmion moves along the direction of the current, now we assume $\mathbf{j}_{\mathrm{hm}}=j_{\mathrm{hm}} \hat{x}$, and the Thiele equation for the AFM skyrmion reads

$$
-\alpha \mathcal{D} \dot{x}+4 \pi \mathcal{B} j_{\mathrm{hm}}-\frac{d V}{d x}=0,
$$

with $\dot{y}=0$. In the same way as in the FM case, the critical current for the AFM skyrmion to overcome a repulsive 


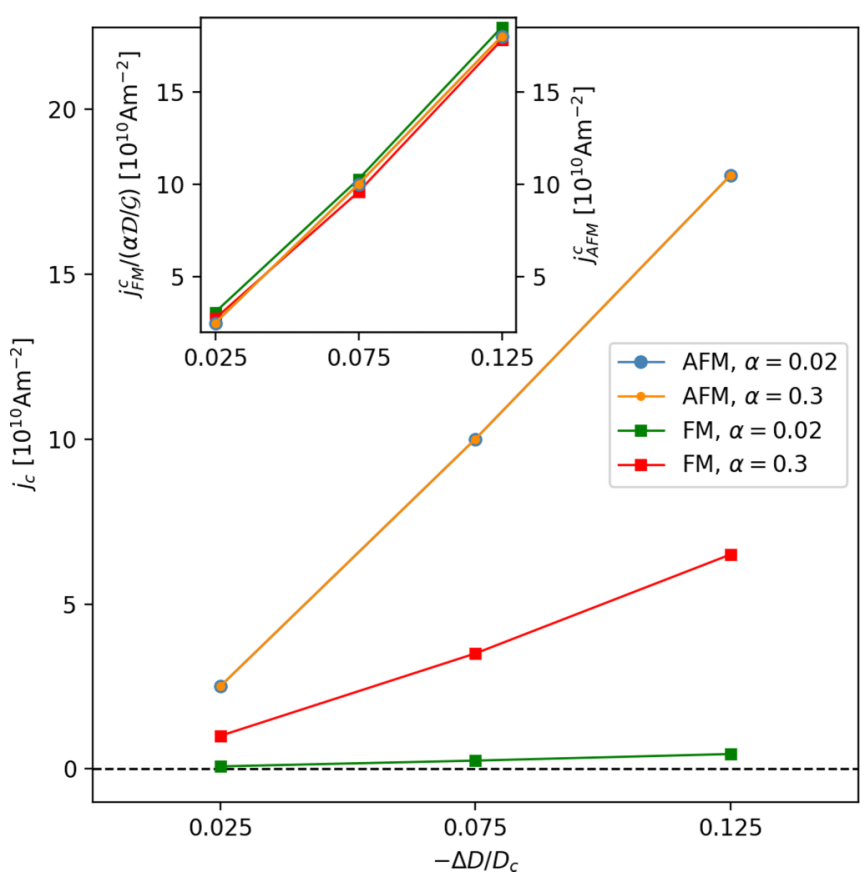

FIG. 9. Critical current for the skyrmion to overcome a heterochiral interface in the AFM and FM cases, as a function of $\Delta D$, with $D_{1}=0.8 D_{c}$ fixed. The inset shows that all data collapse on the same curve with appropriate scaling, following Eq. (17).

potential is obtained by choosing $\dot{x}=0$ in Eq. (16) for the maximal value of $F=d V / d x$, i.e.,

$$
4 \pi \mathcal{B} j_{\mathrm{hm}}^{c}=F_{\max },
$$

which means that the critical current does not depend on $\alpha$ [a similar result is obtained for the CIP scenario if one considers $\mathcal{G}=0$ in Eq. (3)]. Therefore, for $\alpha \ll 1$, not only does the AFM skyrmion travel faster than the corresponding FM one, but the critical current for the AFM skyrmion to overcome the same energy barrier is much higher than that expected for the FM skyrmion [see Eq. (12)]: $j_{\mathrm{AFM}}^{c}=(\mathcal{G} / \mathcal{D} \alpha) j_{\mathrm{FM}}^{c}$. Figure 8 shows a comparison between the AFM and FM skyrmion driven by the CPP scenario in the presence of a DMI interface. The skyrmion is initialized on the left side of the interface, and for the same current density $\left(j=2 \times 10^{10} \mathrm{Am}^{-2}\right)$ the AFM skyrmion moves much faster than the FM one, as shown in Figs. 8(c) and 8(d), but only the FM skyrmion can cross the interface. This means that the enhanced skyrmion confinement reported in ferromagnetic high-DMI racetracks due to spatially engineered DMI [22] is even more effective for the antiferromagnetic racetracks.

Figure 9 shows the numerically calculated critical current for the skyrmion to overcome the heterochiral interface in the AFM and FM cases, as a function of the difference in DMI across the interface. For the FM case, for a lowered damping parameter, the skyrmion moves faster, but the efficiency of the confinement also decreases, which may be a drawback for racetrack applications. However, in the AFM case the critical current is very large for the considered values of $\alpha$, as expected from Eq. (17). In other words, our results indicate that the AFM skyrmion indeed moves faster, yet experiences stronger confinement than the FM skyrmion in heterochiral films, especially for systems with weak damping. Both of these (seemingly contradictory) features establish AFM skyrmions as a favorable choice for skyrmion-based devices. The values of $\alpha$ considered here are similar to those obtained from experimental results on $\mathrm{CoFeB} / \mathrm{W}$ systems [31,45,52] and $\mathrm{Co} /$ Pt layers [29] $(\alpha \approx 0.015$ and $\alpha \approx 0.3$, respectively). The inset in Fig. 9 demonstrates the scaling of the critical current of the FM cases to the AFM results, with factor $(\mathcal{D} \alpha / \mathcal{G})$, as expected from the analytic formulas. Here $\mathcal{G}=4 \pi$ and the dissipative term is calculated from the simulations as specified in Appendix B. For the inset in Fig. 9 we use $\mathcal{D}=4.87 \pi$, calculated for the skyrmion at rest, in the region with $D_{1}=0.8 D_{c}$. Note that similar results can be obtained from the Thiele equation by considering the external potential due to a "boundary" instead of the heterochiral interface (as done in Sec. III A 1).

\section{CONCLUSIONS}

Recent advances in atomically controlled growth of heterostructures have opened the door to heterochiral structures with spatially engineered DMI, with precisely defined interfaces where DMI changes. In this paper, we have addressed the expected behavior of skyrmions in such systems by studying the dynamics of both ferromagnetic and antiferromagnetic skyrmions when encountering a heterochiral interface during their motion. We demonstrated that a local canting of the magnetization, characteristic of the interface where the DMI changes, can strongly deflect the trajectory of a FM skyrmion. We explored the thresholds of this phenomenon both analytically and numerically, and we quantified its dependence on the relevant material parameters. These findings are very useful for the controlled manipulation of either single skyrmions or skyrmion chains in skyrmion-based devices (switches, logic gates, memory elements, to name a few) where, depending on the applied current, one can control which path the skyrmion will take in the corresponding nanoengineered circuit, as exemplified in Sec. III A 3.

In addition, we showed that such a deflection characteristic of the ferromagnetic skyrmion is completely absent in the antiferromagnetic case. Although this finding is detrimental for the applications of the above effect in AFM systems, we demonstrated that the AFM skyrmion holds other advantages over the FM one-it travels much faster for the given applied current, yet it is far better confined in heterochiral films even at high driving currents. This makes AFM skyrmions favorable for skyrmion-based devices in which very fast transfer of information and reliable guidance within specified tracks are essential.

\section{ACKNOWLEDGMENTS}

This work was supported by the Research FoundationFlanders (FWO-Vlaanderen) and Brazilian Agencies FACEPE under Grant No. APQ-0198-1.05/14, CAPES and CNPq.

\section{APPENDIX A: ANTIFERROMAGNETIC MODEL}

The MUMAX ${ }^{3}$ software was originally developed for simulations of ferromagnetic (FM) systems in the continuous field 


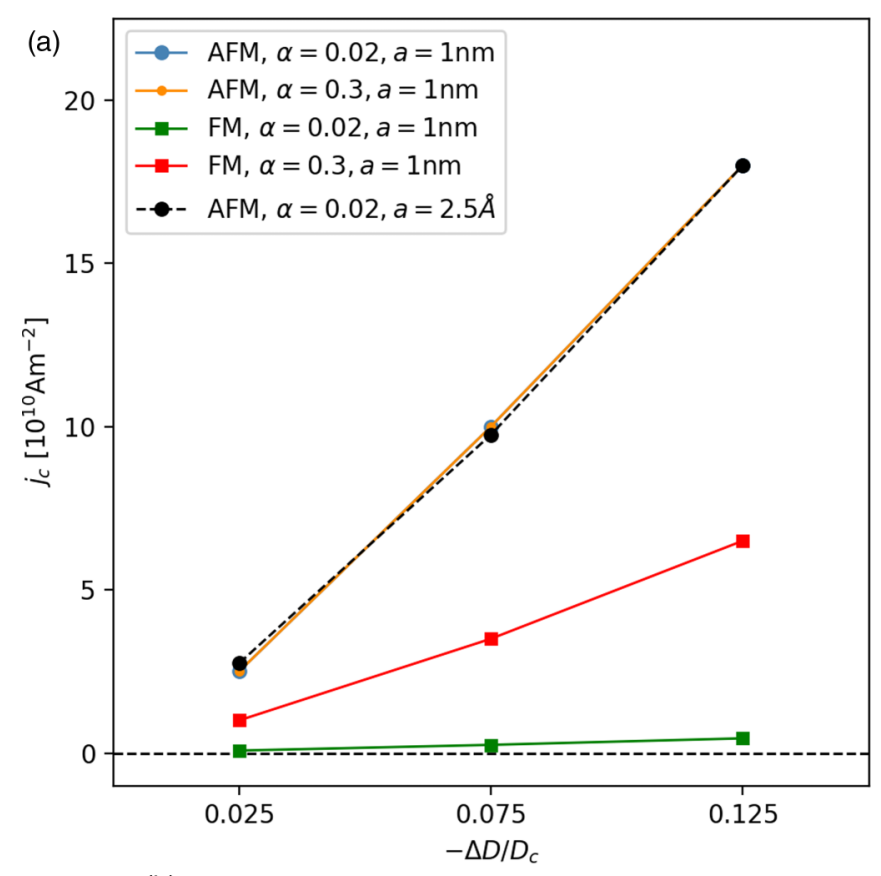

(b)

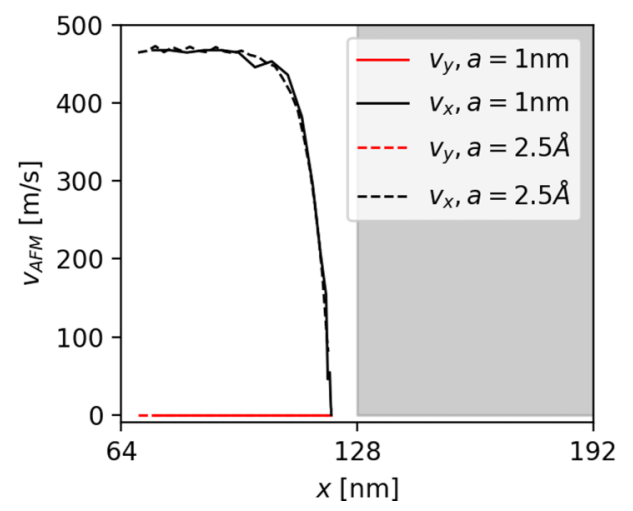

FIG. 10. Comparison of the results obtained in Figs. 9 and 8 of the main text with those obtained for the ultrasmall grid separation of $a=2.5 \AA$. (a) Critical current for the skyrmion to overcome a heterochiral interface in the AFM and FM cases, as a function of $\Delta D$, with $D_{1}=0.8 D_{c}$ fixed. (b) Center-of-mass velocities of the AFM skyrmion during motion shown in Fig. 8(a) of the main text.

approximation. However, in micromagnetic simulations we do not work with a continuous field. Instead we discretize the field on a grid. Therefore, for the case of an antiferromagnetic (AFM) system, which comprises two sublattices of reversely aligned spins, each cell of the mesh grid is now understood as one single spin, and we end up performing an atomistic simulation, with the grid separation now representing the lattice parameter.

The exchange energy density $\mathcal{E}_{\text {ex }}=A\left[\left(\partial_{x} \mathbf{m}\right)^{2}+\left(\partial_{y} \mathbf{m}\right)^{2}\right]$ is the continuous analog form of the classical Heisenberg Hamiltonian $E_{i j}=-J \mathbf{S}^{i} \cdot \mathbf{S}^{j}$, which models the exchange interaction between neighboring spins $\mathbf{S}^{i}$ and $\mathbf{S}^{j}$ in atomistic spin systems. This analogy can be observed by considering, e.g., the first-order finite-difference approximation of the energy density due to variations of the magnetization along the

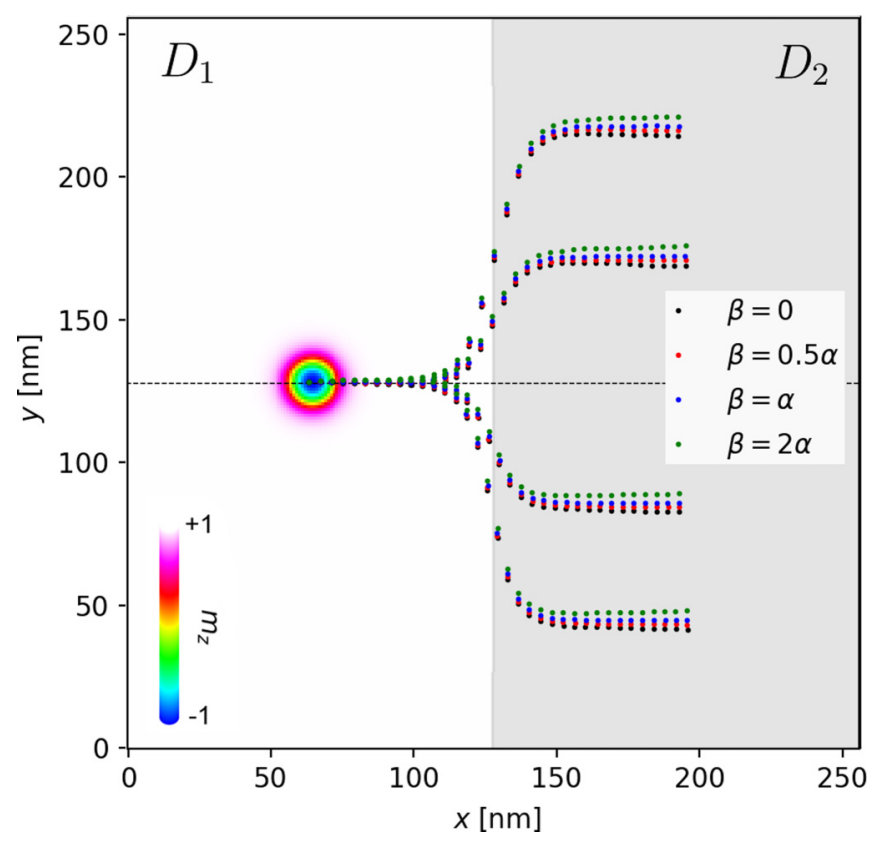

FIG. 11. The skyrmion is initialized on the left side of the diagram. Depending on the difference of the DMI strengths across the interface, $D_{1}$ and $D_{2}$, the skyrmion deflection is positive $(\Delta y>0)$ or negative $(\Delta y<0)$. The dots show the respective trajectories of the skyrmion, and the colors indicate the value of $\beta$ considered, for $j=2 \times 10^{11} \mathrm{~A} \mathrm{~m}^{-2}$ and $\Delta D / D_{c}=0.05,0.025,-0.025$, and -0.05 , respectively, top to bottom.

$x$ direction, i.e.,

$$
A\left(\frac{\mathbf{m}^{i+1}-\mathbf{m}^{i}}{\Delta x}\right)^{2}=\frac{2 A}{(\Delta x)^{2}}\left(1-\mathbf{m}^{i} \cdot \mathbf{m}^{i+1}\right),
$$

where $\mathbf{m}^{i}$ and $\mathbf{m}^{i+1}$ are the magnetizations of adjacent grid cells, with grid separation $\Delta x$ along the $x$ direction. Notice that the energy changes with the dot product of the magnetization in neighboring cells, which is mathematically equivalent to the classical Heisenberg Hamiltonian. Similar to the exchange interaction, the Dzyaloshinskii-Moriya interaction (DMI) $\mathcal{E}_{\mathrm{DMI}}=-D\left[m_{x} \partial_{x} m_{z}-m_{z} \partial_{x} m_{x}+m_{y} \partial_{y} m_{z}-m_{z} \partial_{y} m_{y}\right]$ is the continuous analog of the classical Heisenberg-like Hamiltonian $E_{i j}=\mathbf{D} \cdot\left(\mathbf{S}^{i} \times \mathbf{S}^{j}\right)$, which models the DMI between neighboring spins $\mathbf{S}^{i}$ and $\mathbf{S}^{j}$ with DMI vector D. Again, considering only variations of the magnetization along the $x$ direction, the finite-difference approximation yields

$-D\left[m_{x}^{i} \frac{m_{z}^{i+1}-m_{z}^{i}}{\Delta x}-m_{z}^{i} \frac{m_{x}^{i+1}-m_{x}^{i}}{\Delta x}\right]=\frac{D}{\Delta x} \hat{e}_{y} \cdot\left(\mathbf{m}^{i} \times \mathbf{m}^{i+1}\right)$,

with $\mathbf{m}^{i}$ and $\mathbf{m}^{i+1}$ the magnetizations of adjacent grid cells. This expression is also equivalent to the classical Heisenberg Hamiltonian for the DMI.

Therefore, to perform the atomistic simulation, one needs to consider an ultrasmall mesh grid with grid separation of the order of the atomic distances. To check our results, we reproduced Figs. 8 and 9 of the main text for the antiferromagnetic system, now with a grid separation of $a=2.5 \AA$, which is a typical value for the lattice constant considered in atomistic simulations [50,53]. Figure 10 compares the results of 
Figs. 8 and 9 of the main text with those obtained for the ultrasmall grid separation of $a=2.5 \AA$. Notice that the results do not change considerably by changing the grid separation, which indicates that the AFM simulations presented in the main text can be understood as atomistic simulations on a square lattice.

\section{APPENDIX B: CALCULATION OF THE DISSIPATIVE TENSOR}

The dissipative tensor can be calculated by considering a single magnetic skyrmion with the center located at the origin $r=0$. The components of the dissipative tensor are defined as

$$
\mathcal{D}_{i j}=\int d^{2} r \partial_{i} \mathbf{m} \cdot \partial_{j} \mathbf{m} .
$$

The azimuthal symmetry of the spin configuration leads to $\mathcal{D}_{x x}=\mathcal{D}_{y y}=\mathcal{D}$ and $\mathcal{D}_{x y}=\mathcal{D}_{y x}=0$, and reduces the problem to the $1 \mathrm{D}$ integral

$$
\mathcal{D}=\pi \int_{0}^{\infty} r d r\left[\left(\frac{d \theta(r)}{d r}\right)^{2}+\frac{\sin ^{2} \theta(r)}{r^{2}}\right],
$$

where we used $\mathbf{m}(\mathbf{r})=\sin [\theta(r)] \hat{r}+\cos [\theta(r)] \hat{z}$ in Eq. (B1) for the case of a Néel skyrmion. Here $r=\sqrt{x^{2}+y^{2}}$ is the distance from the the skyrmion core. Equation (B2) can be discretized in the simulation as follows [54]:

$$
\mathcal{D}=\pi \sum_{i=1}^{N}\left[\left(\frac{\theta(i+1)-\theta(i-1)}{2}\right)^{2}+\frac{\sin ^{2} \theta(i)}{i^{2}}\right] \text {, }
$$

where $r=i a$, with $a$ the lattice separation. $N$ is such that $R_{\mathrm{sk}} \ll N a$, with $R_{\mathrm{sk}}$ the skyrmion radius. For the range of parameters considered in this work, we calculate $\mathcal{D} \approx$ $4 \pi-8 \pi$.

\section{APPENDIX C: EFFECTS OF THE NONADIABATIC SPIN TRANSFER TORQUE}

Figure 11 shows the effects of the nonadiabatic spintransfer torque to the skyrmion trajectories with a fixed damping parameter $\alpha=0.02$. The value of $\beta$ does not affect the deflection induced by the interface, but it induces a small constant velocity transverse to the applied current, as expected for a single skyrmion moving in free space.
[1] I. Dzyaloshinsky, A thermodynamic theory of weak ferromagnetism of antiferromagnetics, J. Phys. Chem. Solids 4, 241 (1958).

[2] T. Moriya, Anisotropic superexchange interaction and weak ferromagnetism, Phys. Rev. 120, 91 (1960).

[3] A. Crépieux and C. Lacroix, Dzyaloshinsky-Moriya interactions induced by symmetry breaking at a surface, J. Magn. Magn. Mater. 182, 341 (1998).

[4] A. Bogdanov and A. Hubert, Thermodynamically stable magnetic vortex states in magnetic crystals, J. Magn. Magn. Mater. 138, 255 (1994).

[5] A. N. Bogdanov and U. K. Rößler, Chiral symmetry breaking in magnetic thin films and multilayers, Phys. Rev. Lett. 87, 037203 (2001)

[6] M. Ezawa, Compact merons and skyrmions in thin chiral magnetic films, Phys. Rev. B 83, 100408 (2011).

[7] N. S. Kiselev, A. N. Bogdanov, R. Schäfer, and U. K. Rößler, Chiral skyrmions in thin magnetic films: New objects for magnetic storage technologies? J. Phys. D 44, 392001 (2011).

[8] S. S. P. Parkin, M. Hayashi, and L. Thomas, Magnetic domainwall racetrack memory, Science 320, 190 (2008).

[9] A. Fert, V. Cros, and J. Sampaio, Skyrmions on the track, Nat. Nanotechnol. 8, 152 (2013).

[10] N. Nagaosa and Y. Tokura, Topological properties and dynamics of magnetic skyrmions, Nat. Nanotechnol. 8, 899 (2013).

[11] A. N. Bogdanov and D. A. Yablonskii, Contribution to the theory of inhomogeneous states of magnets in the region of magnetic-field-induced phase transitions. Mixed state of antiferromagnets, Sov. Phys. JETP 69, 142 (1989).

[12] J. Barker and O. A. Tretiakov, Static and Dynamical Properties of Antiferromagnetic Skyrmions in the Presence of Applied Current and Temperature, Phys. Rev. Lett. 116, 147203 (2016).
[13] X. Zhang, Y. Zhou, and M. Ezawa, Antiferromagnetic skyrmion: stability, creation and manipulation, Sci. Rep. 6, 24795 (2016).

[14] H. Xia, C. Jin, C. Song, J. Wang, J. Wang, and Q. Liu, Control and manipulation of antiferromagnetic skyrmions in racetrack, J. Phys. D 50, 505005 (2017).

[15] C. Jin, C. Song, J. Wang, and Q. Liu, Dynamics of antiferromagnetic skyrmion driven by the spin Hall effect, Appl. Phys. Lett. 109, 182404 (2016).

[16] H. D. Rosales, D. C. Cabra, and P. Pujol, Three-sublattice skyrmion crystal in the antiferromagnetic triangular lattice, Phys. Rev. B 92, 214439 (2015).

[17] L. Shen, J. Xia, G. Zhao, X. Zhang, M. Ezawa, O. A. Tretiakov, X. Liu, and Y. Zhou, Dynamics of the antiferromagnetic skyrmion induced by a magnetic anisotropy gradient, Phys. Rev. B 98, 134448 (2018)

[18] S. Rohart and A. Thiaville, Skyrmion confinement in ultrathin film nanostructures in the presence of Dzyaloshinskii-Moriya interaction, Phys. Rev. B 88, 184422 (2013).

[19] J. Mulkers, M. V. Milošević, and B. Van Waeyenberge, Cycloidal versus skyrmionic states in mesoscopic chiral magnets, Phys. Rev. B 93, 214405 (2016).

[20] C. P. Chui, F. Ma, and Y. Zhou, Geometrical and physical conditions for skyrmion stability in a nanowire, AIP Adv. 5, 047141 (2015).

[21] C. Navau, N. Del-Valle, and A. Sanchez, Analytical trajectories of skyrmions in confined geometries: Skyrmionic racetracks and nano-oscillators, Phys. Rev. B 94, 184104 (2016)

[22] J. Mulkers, B. Van Waeyenberge, and M. V. Milošević, Effects of spatially engineered Dzyaloshinskii-Moriya interaction in ferromagnetic films, Phys. Rev. B 95, 144401 (2017). 
[23] D. Stosic, T. B. Ludermir, and M. V. Milošević, Pinning of magnetic skyrmions in a monolayer co film on pt (111): Theoretical characterization and exemplified utilization, Phys. Rev. B 96, 214403 (2017).

[24] D. Stosic, J. Mulkers, B. Van Waeyenberge, T. B. Ludermir, and M. V. Milošević, Paths to collapse for isolated skyrmions in few-monolayer ferromagnetic films, Phys. Rev. B 95, 214418 (2017).

[25] A. L. Balk, K. W. Kim, D. T. Pierce, M. D. Stiles, J. Unguris, and S. M. Stavis, Simultaneous Control of the Dzyaloshinskii-Moriya Interaction and Magnetic Anisotropy in Nanomagnetic Trilayers, Phys. Rev. Lett. 119, 077205 (2017).

[26] A. W. J. Wells, P. M. Shepley, C. H. Marrows, and T. A. Moore, Effect of interfacial intermixing on the Dzyaloshinskii-Moriya interaction in Pt/Co/Pt, Phys. Rev. B 95, 054428 (2017).

[27] A. Vansteenkiste, J. Leliaert, M. Dvornik, M. Helsen, F. GarciaSanchez, and B. Van Waeyenberge, The design and verification of MuMax3, AIP Adv. 4, 107133 (2014).

[28] J. M. D. Coey, Magnetism and Magnetic Materials (Cambridge University Press, Cambridge, 2010).

[29] P. J. Metaxas, J. P. Jamet, A. Mougin, M. Cormier, J. Ferré, V. Baltz, B. Rodmacq, B. Dieny, and R. L. Stamps, Creep and Flow Regimes of Magnetic Domain-Wall Motion in Ultrathin $\mathrm{Pt} / \mathrm{Co} / \mathrm{Pt}$ Films with Perpendicular Anisotropy, Phys. Rev. Lett. 99, 217208 (2007).

[30] J. Sampaio, V. Cros, S. Rohart, A. Thiaville, and A. Fert, Nucleation, stability and current-induced motion of isolated magnetic skyrmions in nanostructures, Nat. Nanotechnol. 8, 839 (2013).

[31] R. Tomasello, E. Martinez, R. Zivieri, L. Torres, M. Carpentieri, and G. Finocchio, A strategy for the design of skyrmion racetrack memories, Sci. Rep. 4, 6784 (2014).

[32] X. Zhang, Y. Zhou, and M. Ezawa, Magnetic bilayer-skyrmions without skyrmion Hall effect, Nat. Commun. 7, 10293 (2016).

[33] W. Jiang, X. Zhang, G. Yu, W. Zhang, X. Wang, M. B. Jungfleisch, J. E. Pearson, X. Cheng, O. Heinonen, K. L. Wang et al., Direct observation of the skyrmion hall effect, Nat. Phys. 13, 162 (2017).

[34] S. Zhang and Z. Li, Roles of Nonequilibrium Conduction Electrons on the Magnetization Dynamics of Ferromagnets, Phys. Rev. Lett. 93, 127204 (2004).

[35] L. Liu, O. J. Lee, T. J. Gudmundsen, D. C. Ralph, and R. A. Buhrman, Current-Induced Switching of Perpendicularly Magnetized Magnetic Layers using Spin Torque from the Spin Hall Effect, Phys. Rev. Lett. 109, 096602 (2012).

[36] N. Perez, L. Torres, and E. Martinez-Vecino, Micromagnetic modeling of Dzyaloshinskii-Moriya interaction in spin Hall effect switching, IEEE Trans. Magn. 50, 1 (2014).

[37] Y. Wang, P. Deorani, X. Qiu, J. H. Kwon, and H. Yang, Determination of intrinsic spin hall angle in pt, Appl. Phys. Lett. 105, 152412 (2014).

[38] J. C. Slonczewski, Current-driven excitation of magnetic multilayers, J. Magn. Magn. Mater. 159, L1 (1996).
[39] J. Xiao, A. Zangwill, and M. D. Stiles, Boltzmann test of Slonczewski's theory of spin-transfer torque, Phys. Rev. B 70, 172405 (2004).

[40] H. V. Gomonay and V. M. Loktev, Spin transfer and currentinduced switching in antiferromagnets, Phys. Rev. B 81, 144427 (2010).

[41] H. V. Gomonay, R. V. Kunitsyn, and V. M. Loktev, Symmetry and the macroscopic dynamics of antiferromagnetic materials in the presence of spin-polarized current, Phys. Rev. B 85, 134446 (2012).

[42] J. Iwasaki, W. Koshibae, and N. Nagaosa, Colossal spin transfer torque effect on skyrmion along the edge, Nano Lett. 14, 4432 (2014).

[43] S. Mizukami, Y. Ando, and T. Miyazaki, The study on ferromagnetic resonance linewidth for $\mathrm{NM} / 80 \mathrm{NiFe} / \mathrm{NM}(\mathrm{NM}=\mathrm{Cu}$, Ta, Pd and Pt) films, Jpn. J. Appl. Phys. 40, 580 (2001).

[44] M. Oogane, T. Wakitani, S. Yakata, R. Yilgin, Y. Ando, A. Sakuma, and T. Miyazaki, Magnetic damping in ferromagnetic thin films, Jpn. J. Appl. Phys. 45, 3889 (2006).

[45] S. Ikeda, K. Miura, H. Yamamoto, K. Mizunuma, H. D. Gan, M. Endo, S. Kanai, J. Hayakawa, F. Matsukura, and H. Ohno, A perpendicular-anisotropy $\mathrm{CoFeB}-\mathrm{MgO}$ magnetic tunnel junction, Nat. Mater. 9, 721 (2010).

[46] M. Oogane, T. Kubota, Y. Kota, S. Mizukami, H. Naganuma, A. Sakuma, and Y. Ando, Gilbert magnetic damping constant of epitaxially grown co-based heusler alloy thin films, Appl. Phys. Lett. 96, 252501 (2010).

[47] See Supplemental Material at http://link.aps.org/supplemental/ 10.1103/PhysRevB.99.104409 for the animation of a skyrmion chain in a nanoengineered circuit manipulated by pulsed current, corresponding to Fig. 6 .

[48] X. Zhang, M. Ezawa, and Y. Zhou, Magnetic skyrmion logic gates: conversion, duplication and merging of skyrmions, Sci. Rep. 5, 9400 (2015).

[49] A. N. Bogdanov, U. K. Rößler, M. Wolf, and K.-H. Müller, Magnetic structures and reorientation transitions in noncentrosymmetric uniaxial antiferromagnets, Phys. Rev. B 66, 214410 (2002).

[50] R. Khoshlahni, A. Qaiumzadeh, A. Bergman, and A. Brataas, Ultrafast generation and dynamics of isolated skyrmions in antiferromagnetic insulators, Phys. Rev. B 99, 054423 (2019).

[51] P. F. Bessarab, D. Yudin, D. R. Gulevich, P. Wadley, M. Titov, and $\mathrm{O}$. A. Tretiakov, Stability and lifetime of antiferromagnetic skyrmions, arXiv:1709.04454.

[52] C. F. Pai, L. Liu, Y. Li, H. W. Tseng, D. C. Ralph, and R. A. Buhrman, Spin transfer torque devices utilizing the giant spin Hall effect of tungsten, Appl. Phys. Lett. 101, 122404 (2012).

[53] R. F. L. Evans, W. J. Fan, P. Chureemart, T. A. Ostler, M. O. A. Ellis, and R. W. Chantrell, Atomistic spin model simulations of magnetic nanomaterials, J. Phys.: Condens. Matter 26, 103202 (2014).

[54] J. Iwasaki, M. Mochizuki, and N. Nagaosa, Universal currentvelocity relation of skyrmion motion in chiral magnets, Nat. Commun. 4, 1463 (2013). 